Original Article

\title{
Frequency of Radix Entomolaris/ Paramolaris in Permanent Mandibular First Molars of Patients Visiting Hamdard University Dental Hospital
}

\author{
Syed Abrar Ali, Mehmood Hussain, Muhammad Shahzad, Ahmed Tariq
}

\begin{abstract}
OBJECTIVE: To see the prevalence of an additional root in permanent mandibular first molars in patients presenting for dental checkup for root canal treatment.

METHODOLOGY: This observational study was carried out at Department of Endodontics, Hamdard University Dental Hospital, Karachi from June 2016 to May 2017. Sample size was Eight hundred and three permanent mandibular first molars from 568 females and 235 males were examined over a period of 1 year. Presence of a third root was revealed by diagnostic periapical radiographs and the comparison between males and females and between the right and left sides of the mandible were recorded.

RESULTS: Out of the $\mathbf{8 0 3}$ permanent mandibular first molars that were observed, only 17 were found to have an accessory root. Out of total 803 samples that were being evaluated, only 17 subjects were found to have an extra root with a prevalence of $2.12 \%$. The incidence of extra root was found to be higher in males.
\end{abstract}

CONCLUSION: Though the incidence of radix entomolaris \& paramolaris observed among the examined population was quite low, detection of radix is crucial to a successful endodontic treatment. More in depth study and detection methods are required for better identification of this unique anomaly.

KEYWORDS: Radix, Entomolaris, Paramolaris, Permanent Mandibular, First Molars.

This article may be cited as: Ali SA, Hussain M, Shahzad M, Tariq A. Frequency of Radix Entomolaris/ Paramolaris in Permanent Mandibular First Molars of Patients Visiting Hamdard University Dental Hospital. J Liaquat Uni Med Health Sci. 2019;18(02):125-8. doi: $10.22442 /$ jumhs.191820614

\section{INTRODUCTION}

Healing of a periapical infection and the success of an endodontic treatment depends on thorough cleaning and shaping of root canals ${ }^{1}$.

A detailed understanding, knowledge of the morphology and internal tooth anatomy is necessary for a tooth undergoing root canal treatment. A clinician should be able to anticipate any anatomical alterations that may be present, because some endodontic treatment failures occur as a result of lack of identifying these alterations ${ }^{2}$.

According to the literature, permanent mandibular first molarsshowvarious anatomical variations and endodontic treatment of these particular teeth is quite difficult ${ }^{3}$.

Most of the time, permanent mandibular first molars have two roots, a mesial and a distal root ${ }^{1}$. Variations in the number of roots as well as the internal canal configuration in mandibular molars are not unheard of (1), including additional roots on the distolingual aspect, which is named as Radix Entomolaris (RE) or on the mesiobuccal side, which is named the Radix Paramolaris (RP) ${ }^{4}$. The distolingual root is usually smaller than the additional mesiobuccal root and exhibits a curve in buccolingual direction ${ }^{5}$.

A few theories regarding the origin of RE and RP have been put forward. One states that a radix may arise due to external factors during tooth development, whereas another indicates that a gene mutation (atavism) is responsible for it. However the later theory is one that is more accepted ${ }^{6}$.

Knowledge of incidence, presence and position of any anatomical variation is important as it has significance in clinical dentistry and treatment outcomes. Epidemiological studies have shown that it is important to be cautious about presence of accessory roots while performing root canal treatment on permanent mandibular first molars, however the data is rare in relevance to Karachi population ${ }^{7}$. The reason for this study was to discover the percentage of permanent mandibular first molar teeth having three 
roots in a Pakistani population using conventional radiographs.

\section{METHODOLOGY}

The study was conducted in the department of Operative Dentistry, faculty of dental sciences, Hamdard University Dental Hospital, Hamdard College of Medicine and Dentistry, Karachi, Pakistan. The study design was of observational type and data collection method was done by convenient sampling technique.

Ethical permission for the study from university was taken prior to study and after obtaining consent, in total 803 patients, 235 male patients and 568 female patients, above the age of 18 years were screened from June 2016 to June 2017. A subject was required to have at least one permanent mandibular first molar to be included in study. Age, sex and race of the subjects were recorded.

About 803 mandibular first molars were evaluated using periapical radiographs at $30^{\circ}$ mesial angulation. The radiographs were developed and fixed using standard procedure as per American Dental Association specifications ${ }^{10}$, followed by independent evaluation by two trained observers. The intra observer reliability was checked by kappa statistics, where $\mathrm{k}=1$.

The radiograph was obtained by $\mathrm{A} 60 \mathrm{KV}$, PROX Wireless Portable X-ray unit. The exposure time was set at 0.8 seconds. RDX-58 E(Primax; Berlin, Germany) soft film was used, positioned in a film holding device. Visual method was used for radiograph development.

The criteria used to identify the presence of a radix were a clearly visible extra root, originating from the root trunk, indicated by the presence of the pulp and periodontal space radiolucency.

The descriptive statistics of incidence and occurrence of an extra root were then analyzed using SPSS software version 23 .

Inclusion Criteria was Patients aged 18 years and above, Patients with erupted permanent mandibular first molars,Permanent mandibular first molars with an additional third root evident on radiograph.

Exclusion Criteria was Patients below 18 years of age, Patients with missing permanent mandibular first molars, Permanent mandibular first molars with two roots only evident on periapical radiograph.

Operational Definitions: Radix: an additional or accessory root, Paramolaris: accessory root located on the buccal aspect, Entomolaris: accessory root located on lingual aspect.

\section{RESULTS}

A total of 803 subjects comprising of 568 females and 235 males formed the study sample. Out of total 803 samples that were being evaluated, only 17 subjects were found to have an extra root with a prevalence of $2.12 \%$. The incidence of extra root was found to be higher in males.

Out of total 568 females, only 10 subjects were found to have an extra root while out of 235 male subjects that were being evaluated, only 7 subjects were found to have an extra root showing prevalence of $1.76 \%$ and $2.97 \%$ respectively.

TABLE I: NUMBER AND PERCENTAGE OF RADIX IN PERMANENT MANDIBULAR FIRST MOLAR

\begin{tabular}{|l|c|c|c|c|}
\hline $\begin{array}{c}\text { Gender } \\
\text { Ratio }\end{array}$ & $\begin{array}{c}\text { Number } \\
\text { of } \\
\text { Patients }\end{array}$ & $\begin{array}{c}\text { Number of } \\
\text { Radix (\%) }\end{array}$ & $\begin{array}{c}\text { Right First } \\
\text { Mandibular } \\
\text { Molars with } \\
\text { radix (\%) }\end{array}$ & $\begin{array}{c}\text { Left First } \\
\text { Mandibular } \\
\text { Molars with } \\
\text { radix (\%) }\end{array}$ \\
\hline Female & 568 & $10(1.76 \%)$ & $03(30 \%)$ & $07(70 \%)$ \\
\hline Male & 235 & $07(2.97 \%)$ & $02(28.6 \%)$ & $05(71.4 \%)$ \\
\hline Total & 803 & $17(2.12 \%)$ & $05(29.4 \%)$ & $12(70.6 \%)$ \\
\hline
\end{tabular}

\section{DISCUSSION}

Presence of a radix requires that a clinician be careful in carrying out root canal treatment of the permanent lower molars. Visual inspection of the clinical crown can aid in the identification of an additional root since it is sometimes associated with additional number of cusps or root canals or lobe ${ }^{1}$.

Careful evaluation of diagnostic radiograph plays an important role in planning an endodontic treatment ${ }^{1,5}$. Radiographs at varying angulation should be taken and assessed ${ }^{8}$. When the outline of the root canals of distal root seems unclear on a radiograph then a third hidden root should be suspected. For confirmation, various literature suggests that a second radiograph with 30 degree mesial or distal angulation should be taken which can help in revealing the presence of a radix ${ }^{4}$.

Various studies were conducted to show the prevalence of radix entamolaris or paramolaris. Mukhaimer R $2014^{1}$ reported the frequency as 3.73\% in Palestinian population(1), $3.10 \%$ prevalence was reported by Rahimi $\mathrm{S}$ et $\mathrm{al}^{5}$ in an Iranian population, 
$8.3 \%$ were reported in Indian population ${ }^{9}$, Calberson FL $2007^{10}$ reported $5 \%$ in Mongolian race, which is slightly greater than those of Caucasian, African \& Asian populations. Kuzekanani M $2017^{4}$ found that in Iranian population there was a prevalence of $6 \%$ of Radix in first molars while in second molars it was less than $1 \%$.

Mukhaimer R $2014^{1}$ found no significant difference between females and males regarding the incidence of three rooted permanent mandibular molars as opposed to our study which concludes that radix entomolaris or paramolaris is more prevalent in female gender which was in accordance with the study by Rahimi $S$ et $\mathrm{al}^{5}$.

Advanced diagnostic tools like dental operating microscope (DOM) and cone-beam computed tomography (CBCT) have been proven to have greater significance over traditional diagnostic tools by various researchers. СВCT enables the clinician to identify canal morphology, numbers of canals, and relative positioning of the additional root $^{11}$.

A limitation of the current study is that data was only collected from one center in Karachi and coupled with the use of single horizontally angled two dimensional periapical radiograph. Cone beam computed tomographyovercomes these drawbacks by reducing superimposition and permitting better view of threedimensional structures. The discrepancies between the results of the present study and other studies might be attributed to ethnic, racial and method differences.

Though the incidence of radix entomolaris \& paramolaris observed among the examined population was quite low, detection of radix is crucial to a successful endodontic treatment. More in depth study and detection methods are required for better identification of this unique anomaly

\section{CONCLUSION}

Though the incidence of radix entomolaris \& paramolaris observed among the examined population was quite low, detection of radix is crucial to a successful endodontic treatment. More in depth study and detection methods are required for better identification of this unique anomaly

Ethical Permission: ERC Reference No. HCM\&D/ HUDH/2018/142 dated: 30-03-2018.

Conflict of interest: There is no conflict of interest.
Funding: There was no any funding agency.

\section{REFERENCES}

1. Mukhaimer R, Azizi Z. Incidence of radix entomolaris in mandibular first molars in Palestinian population: a clinical investigation. Int Scholar Res Notices. 2014; 1-5. doi:10.1155/2014/405601

2. López-Rosales $E$, Castelo-Baz $P$, De Moor R, Ruíz-Piñón $M$, Martín-Biedma $B$, Varela-Patiño $P$. Unusual root morphology in second mandibular molar with a radix entomolaris, and comparison between cone-beam computed tomography and digital periapical radiography: a case report. J Med Case Rep. 2015; 9(1): 201.doi:10.1186/ s13256-015-0681-x

3. Sarangi P, Uppin VM. Mandibular First Molar with a Radix Entomolaris: An Endodontic Dilemma. J Dent(Tehran). 2014; 11(1): 118-22.

4. Kuzekanani M, Walsh LJ, Haghani J, Kermani AZ. Radix Entomolaris in the Mandibular Molar Teeth of an Iranian Population. Int J Dent. 2017; 1-4. doi:10.1155/2017/9364963

5. Rahimi S, Mokhtari H, Ranjkesh B, Johari M, Reyhani MF, Shahi S, et al. Prevalence of Extra Roots in Permanent Mandibular First Molars in Iranian Population: A CBCT Analysis. Iran Endod J. 2017; 12(1): 70-73._doi: 10.22037/iej.2017.14

6. Różyło TK, Piskórz MJ, Różyło-Kalinowska IK. Radiographic appearance and clinical implications of the presence of radix entomolaris and radix paramolaris. Folia Morphol(Warsz). 2014; 73(4): 449-54. doi: 10.5603/FM.2014.0067.

7. Faraz SA, Tariq A, Jameel A. Root Canal Morphology of Mandibular First Permanent Molars -Karachi Sample. Pak Oral Dent J. 2015; 35(2): 294-98.

8. Souza-Flamini LE, Leoni GB, Chaves JF, Versiani MA, Cruz-Filho AM, Pécora JD, et al. The Radix Entomolaris and Paramolaris: A Micro-Computed Tomographic Study of 3-rooted Mandibular First Molars. J Endod. 2014; 40(10): 1616-21. doi: 10.1016/j.joen.2014.03.012.

9. Gupta A, Duhan J, Wadhwa J. Prevalence of three rooted permanent mandibular first molars in Haryana (North Indian) population. Contemp Clin Dent. 2017; 8(1): 38-41.doi: 10.4103/ccd.ccd_6 9 9_16

10. Calberson FL, De Moor RJ, Deroose CA. The radix entomolaris and paramolaris: clinical 
approach in endodontics. J Endod. 2007; 33(1): 58-63.

11. Kamble AP, Pawar RR, Mattigatti S, Mangala T, Makandar S. Cone-beam computed tomography as advanced diagnostic aid in endodontic treatment of molars with multiple canals: Two case reports. J Conserv Dent. 2017; 20(4): 27377.doi: 10.4103/0972-0707.219194

AUTHOR AFFILIATION:

Dr. Syed Abrar Ali (Corresponding Author) Associate Professor, Department of Operative Hamdard University Dental Hospital Hamdard College of Medicine and Dentistry Hamdard University, Karachi, Sindh-Pakistan. Email: dentist4@hotmail.com

\section{Dr. Mehmood Hussain}

Associate Professor, Department of Prosthodontics Hamdard University Dental Hospital

Hamdard College of Medicine and Dentistry

Hamdard University, Karachi, Sindh-Pakistan.

\section{Dr. Muhammad Shahzad}

Associate Professor Department of Oral Maxillofacial Surgery Liaquat University of Medical and Health Sciences Jamshoro, Sindh-Pakistan.

\section{Dr. Ahmed Tariq}

F.C.P.S II Resident, Department of Operative Hamdard University Dental Hospital

Hamdard College of Medicine and Dentistry

Hamdard University, Karachi, Sindh-Pakistan. 